\title{
MAGNETIC AND CRYSTALLOGRAPHIC CHARACTERISTICS OF Co-Cr LAYERS WITH Al ULTRA-THIN INTERLAYERS
}

\author{
Takakazu TAKAHASHI*, Nagayasu IKEDA* and Masahiko NAOE**
}

* Dept. of Electronic \& Computer Engineering, Toyama University, Toyama-shi,

Toyama 930, JAPAN

* Dept. of Physical Electronics, Tokyo Institute of Technology, Meguro-ku, Tokyo 152, JAPAN

Abstracts--- $\mathrm{Co}_{81} \mathrm{Cr}_{19} / \mathrm{Al}$ multilayered films with thicknesses of $\mathrm{Co}_{81} \mathrm{Cr}_{19}$ layers in the range of $46 \sim 170 \AA$ and $A l$ ones in the range of $7 \AA \sim 14 \AA$ have been prepared on glass-slide substrate at room temperature by using the facing targets sputtering (FTS) apparatus. The dependence of the magnetic and crystallographic characteristics on the thicknesses of $\mathrm{Co}-\mathrm{Cr}$ layers and $\mathrm{Al}$ ones have been investigated. The magnetoresistance effect of the specimen films have been also examined. The intrinsic $\mathrm{Co}_{81} \mathrm{Cr}_{19}$ layers were composed of hcp phase crystallites with well orientation of the c-axis normal to the film plane. Both the crystallinity of $\mathrm{Co}_{81} \mathrm{Cr}_{19}$ layers was improved and the d-spacing between the c-planes was expanded by using the Al interlayers. The saturation magnetization, the coercivity, and the in-plane squareness ratio depended strongly on the thicknesses of $\mathrm{C}_{81} \mathrm{Cr}_{19}$ layers and $\mathrm{Al}$ ones. The magnetic anisotropy can be controlled by the thickness of Al layer. The magnetoresistivity ratio was negative and its absolute value was as small as $0.05 \%$ in $14 \AA-A l$ layer.

\section{INTRODUCTION}

The multilayered films composed of ferromagnetic layers and paramagnetic ones has developed rapidly and its achievements have become extremely attractive, since it is expected that the strong magnetic interactions at the interfaces between both of layers may occur. [1-4]

Co-Cr films have been extensively studied, in particular, for perpendicular magnetic recording media. However, the magnetic properties was not yet controlled in the films with single layer of $\mathrm{Co}-\mathrm{Cr}$ alloy because the origin of magnetic anisotropy perpendicular to the film plane was not yet made enough clear. Therefore, it is necessary to propose a new type of medium in order to control the saturation magnetization, the coercivity and the magnetically anisotropic direction.

In this study, the $\mathrm{Co}-\mathrm{Cr} / \mathrm{Al}$ multilayered films composed of $\mathrm{Co}-\mathrm{Cr}$ and $\mathrm{Al}$ layers have been prepared by using the facing targets sputtering (FTS) apparatus $[1,5]$ with two pairs of targets. The dependence of the magnetic and crystallographic characteristics of the films on the thickness of $\mathrm{Co}-\mathrm{Cr}$ and $\mathrm{Al}$ layers have been investigated in detail.
The magnetoresistance effect of the films have been also examined with high accuracy.

\section{EXPERIMENTAL PROCEDURE}

Figure 1 shows the schematic diagram of the FTS apparatus for preparing multilayerd films. Multilayered films have been prepared by moving substrate alternately

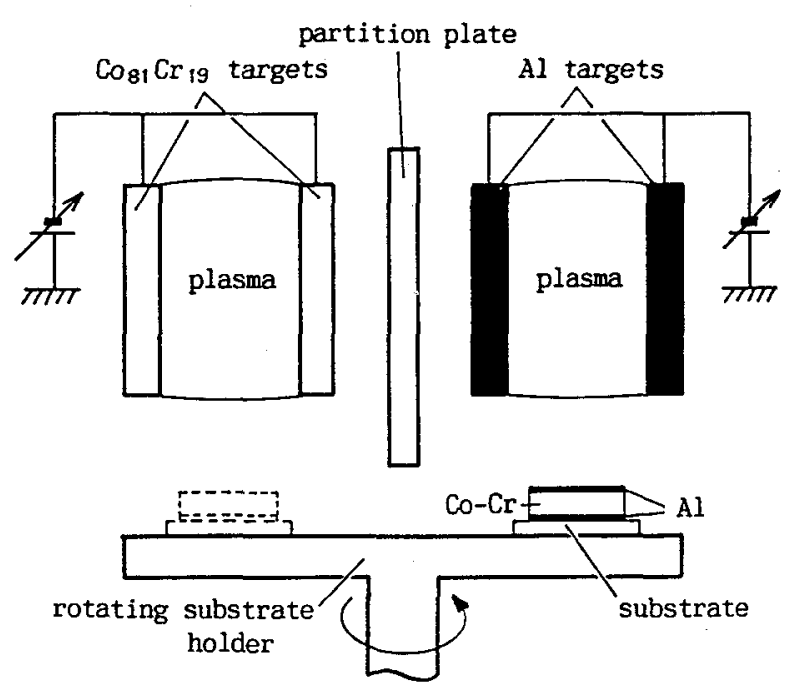

Fig.1. FTS apparatus for preparing multilayered films. 


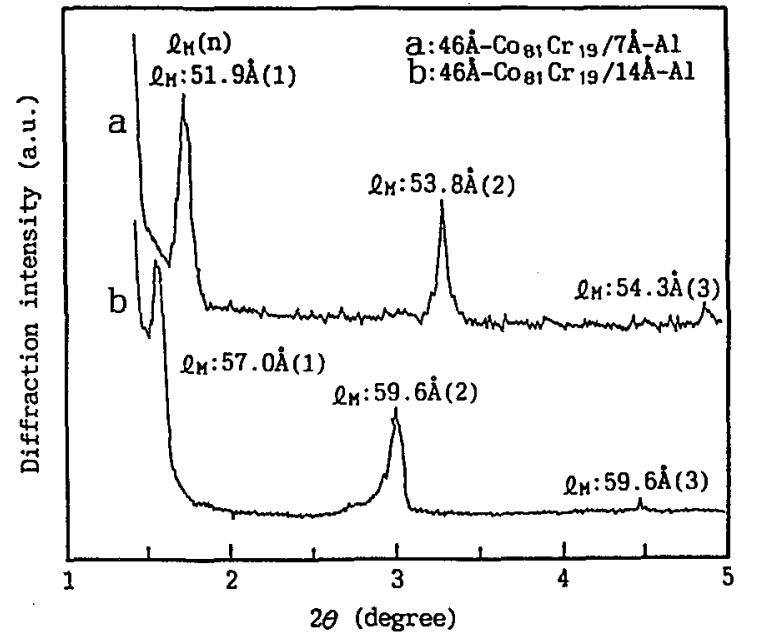

Fig.2. Small angle X-ray diffraction diagrams of the films with modulation periods of $46 \AA-\mathrm{Co}_{81} \mathrm{Cr}_{19} / 7 \AA-\mathrm{Al}$ and $46 \AA-\mathrm{Co}_{81} \mathrm{Cr}_{19} / 14 \AA-A l$.

between two locations to deposit $\mathrm{Co}-\mathrm{Cr}$ and Al layers. The rectangular targets of $\mathrm{Co}_{81} \mathrm{Cr}_{19}$ alloy and pure Al with the purity of 99.9 and $99.999 \%$, respectively, were used, of which the length, width and thickness were $16,10,0.5 \mathrm{~cm}$, respectively. The background pressure was less than $1.2 \times 10^{-6}$ Torr. Ar gas with the purity of $99.999 \%$ was introduced into the chamber at the pressure of $0.5 \mathrm{mTorr}$. The $\mathrm{Co}_{81} \mathrm{Cr}_{19} / \mathrm{Al}$ multilayered films have been prepared by depositing alternately $\mathrm{Co}_{81} \mathrm{Cr}_{19}$ and $\mathrm{Al}$ layers on glass-slide substrates. Deposition rates of $\mathrm{Co}_{81} \mathrm{Cr}_{19}$ and $\mathrm{Al}$ layers at DC discharge current of $1 \mathrm{~A}$ were 6.5 and $7 \AA / \mathrm{sec}$ at applied voltage of 520 and of $470 \mathrm{~V}$, respectively. The thickness of $\mathrm{Co}-\mathrm{Cr}$ layers $\ell_{C_{0}-C_{r}}$ was varied in the range of $46 \sim 170 \AA$, while, the thicknesses of Al layer $\ell_{A I}$ were 7 and $14 \AA$. The total film thickness $\sum\left(\ell_{c_{0}-C_{r}}+l_{A 1}\right)$ was about $1500 \AA$. The first and last layers were $A l$ ones so that every $\mathrm{Co}_{81} \mathrm{Cr}_{19}$ layer was sandwiched by Al ones.

For the purpose of investigating the effects of Al layers in detail, the two types of specimen films with $\ell_{\mathrm{c}_{0}-\mathrm{C}_{\mathrm{r}}}$ of $46 \AA$ were prepared by intermittent sputtering method, where one was the films A composed of intermittently deposited $\mathrm{Co}_{81} \mathrm{Cr}_{19}$ multilayers and another was the films $B$ with the $\mathrm{Co}_{81} \mathrm{Cr}_{19}$ multilayers sandwiched by only the first and last layers of $\mathrm{Al}$.

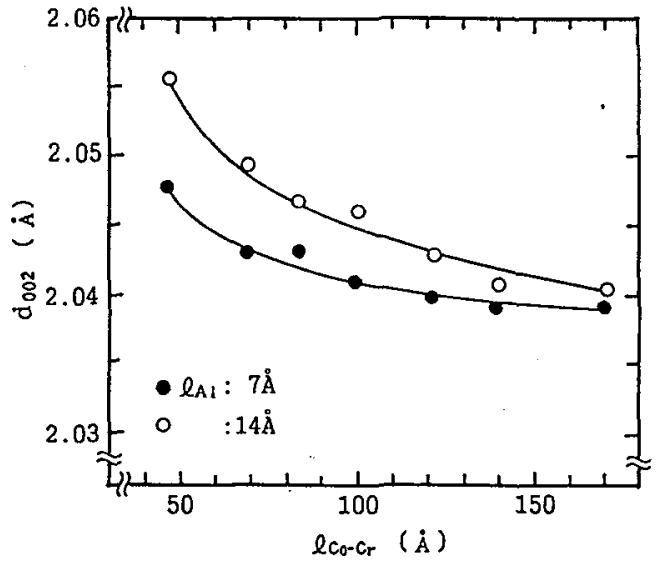

Fig. 3. Dependence of interplanar spacing of (002) $d_{002}$ on thickness of $\mathrm{Co}_{81} \mathrm{Cr}_{19}$ layer $\ell \mathrm{c}_{0}-\mathrm{C}_{r}$.

The crystal structure was analyzed by $X$-ray diffractometry. The saturation magnetization and the coercivity at room temperarure were measured on the hysteresis loops by means of VSM. The anisotropy field $H k$ was estimated on the loops in the direction of the hard magnetization. Magnetoresistivity was measured with a four point probe at room temperature.

\section{RESULTS AND DISCUSSION}

The composition of the deposited $\mathrm{Co}-\mathrm{Cr}$ layers was confirmed to be almost equal to $\mathrm{Co}_{81} \mathrm{Cr}_{19}$, that of target alloy by EPMA.

Figure 2 shows the typical small angle $\mathrm{X}$-ray diffraction diagrams of two kinds of specimen films with modulation periods of $46 \AA-\mathrm{Co}_{81} \mathrm{Cr}_{19} / 7 \AA-A l$ and $46 \AA-\mathrm{Co}_{81} \mathrm{Cr}_{19} / 14 \AA-\mathrm{Al}$, respectively. The three peaks of the both of films were clearly observed. Their modulation lengths $\ell_{M}$ calculated from the angles at the position of these peaks were in good accordance with the expected ones. This may imply that the good periodic structure could be formed in the films deposited by using FTS apparatus. The $\mathrm{Co}_{81} \mathrm{Cr}_{19}$ layers in all specimen were composed intrinsically of the hcp phase crystallites with well orientation of c-axis normal to the film plane.

Figure 3 shows the dependence of the interplanar spacing between (002) planes $\mathrm{d}_{002}$ of hcp Co-Cr crystallites in multilayered films on $l c_{0}-c_{r}$. Both of $d_{002}$ were expanded gradually with decrease of 


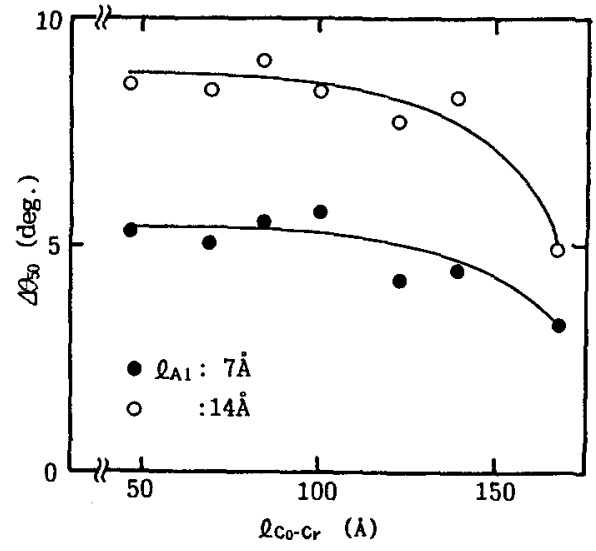

Fig.4. Dependence of c-axis dispersion $\Delta \theta_{50}$ on $l c_{0}-c_{r}$.

$\ell c_{0}-c_{r}$, and was about $2.056 \AA$ at $\ell c_{0}-C_{r}$ of 46 $\AA$ and $\ell_{A 1}$ of $14 \AA$. These values of $\mathrm{d}_{002}$ were longer than that of $\mathrm{Co}_{81} \mathrm{Cr}_{19}$ single layer films $(2.035 \AA)$.[6] The specimen film $A$ and $B$ exhibited $\mathrm{d}_{002}$ of 2.038 and $2.037 \AA$, respectively. This may be due to the difference in Young's modulus between $\mathrm{Co}-\mathrm{Cr}$ alloy and Al. Al is more easily expanded than $\mathrm{Co}-\mathrm{Cr} . \mathrm{d}_{002}$ in $14 \AA-\mathrm{Al}$ layers are longer than that in $7 \hat{\AA}-\mathrm{Al}$ ones. This may be due to that the compressive stress depends on $\ell_{\mathrm{Al}}$.

Figure 4 shows the dependence of the dispersion of c-axis orientation $\Delta \theta_{50}$ in multilayered films on $\ell c_{0}-c_{r}$. Both of $\Delta A_{50}$ decreased gradually with increasing $\ell_{\mathrm{c}_{0}-\mathrm{C}_{r}}$. The crystallinity was improved as $l c_{0}-c_{r}$ increased. The specimen film $A$ and $B$ exhibited $A_{50}$ of 9.6 and $11^{\circ}$, respectively. $\Delta \Theta_{50}$ in $7 \AA-A l$ layers was smaller than that in $14 \AA-A l$ ones. This may imply that $\mathrm{Co}-\mathrm{Cr}$ crystallites may grow epitaxially in $7 \AA-A l$ layers. On the ohter hand, Co-Cr crystallites in $14 \AA-A l$ layers may not grow epitaxially but their grains may become fine by large compressive stress. It has been found from Figs. 3 and 4 that the crystallinity was improved and the c-axis was expanded by using the Al interlayers.

The easy direction of magnetization in the films was perpendicular to the film plane in $7 \AA-A l$ layers.

Figure 5 shows the dependence of the saturation magnetization $M s$ on $l c_{0}-C_{r}$. Ms were calculated for $\Sigma\left(\ell_{C_{0}-C_{r}}+\ell_{A 1}\right)$. Both Ms depended significantly on $l c_{0}-c_{r}$. Ms took maximum value of $610 \mathrm{emu} / \mathrm{cc}$ at $\ell \mathrm{c}_{0}-\mathrm{C}_{r}$ of $84 \AA$

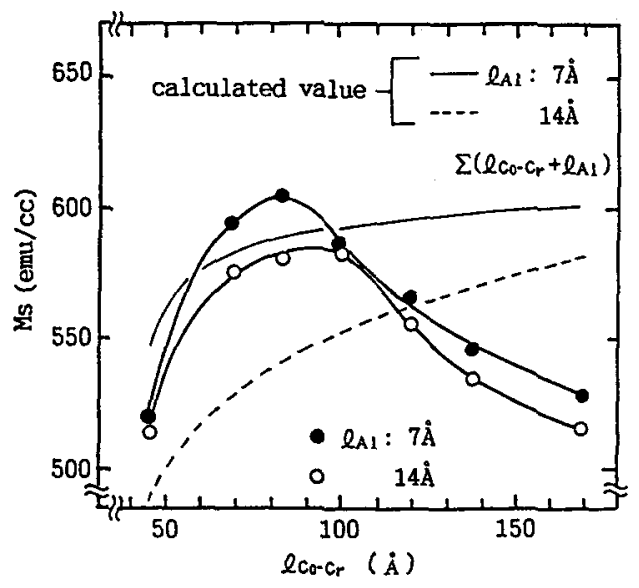

Fig.5. Dependence of saturation magnetization Ms on $\ell c_{0}-C_{r}$.

in $7 \AA-A l$ layers. Ms of the specimen film $A$ and $B$ are 630 , and $640 \mathrm{emu} / \mathrm{cc}$, respectively, being larger than that of the $\mathrm{Co}_{81} \mathrm{Cr}_{19} / \mathrm{Al}$ multilayered films with $l \mathrm{C}_{0}-\mathrm{Cr}_{r}$ of $46 \AA$. The calculated Ms based on the experimental results of $\mathrm{Co}-\mathrm{Cr}$ films by intermittent deposition, where $\ell_{A 1}$ is taken account in. Ms in $\mathrm{Co}_{81} \mathrm{Cr}_{19} / \mathrm{Al}$ multilayered films are strongly different from the calculated ones. This may be caused to the segregation of chromium by large compressive stress occurred in the plane of $\mathrm{Co}_{81} \mathrm{Cr}_{19}$ layers. This consideration may be supported by the significant extention of $\mathrm{d}_{002}$ as shown in Fig. 3 .

Figure 6 shows the dependence of the parallel coercivity $\mathrm{Hc}_{j}$ and the perpendicular one $\mathrm{Hc}_{\perp}$ to the film plane on l $\mathrm{C}_{0}-\mathrm{CC}_{r}$. Both of $\mathrm{Hc}_{\mu}$ and $\mathrm{Hc}_{\perp}$ also depended on $\ell c_{0}-C_{r} . \quad H c_{\perp}$ of the specimen film $A$ and $B$ are 400 , and 3500 e, respectively, being larger than that of the $\mathrm{Co}_{81} \mathrm{Cr}_{19} / \mathrm{Al}$ multilayered films with $\ell c_{0}-c_{r}$ of $46 \AA$. In $7 \AA-A l$ layers, $\mathrm{Hc}_{\perp}$ is higher in the range of $70 \sim 140 \AA$, where $\mathrm{Co}-\mathrm{Cr}$ grains may become fine by compressive stress. On the other hand, in $14 \AA-A l$ layers, $H c$ increased gradually with increasing $\ell \mathrm{c}_{0}-\mathrm{C}_{r}$. $\mathrm{HC}$ also depend on $\ell_{A 1}$. This suggests that the multilayered films with $7 \AA$-Al layers have some effect at the interface between $\mathrm{Al}$ and $\mathrm{Co}-\mathrm{Cr}$ layers.

Figure 7 shows the dependence of the in-plane squareness ratio $(\mathrm{Mr} / \mathrm{Ms})_{\|}$on

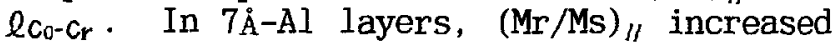
rapidly with increasing $\ell c_{0}-C_{r}$ from 46 to 


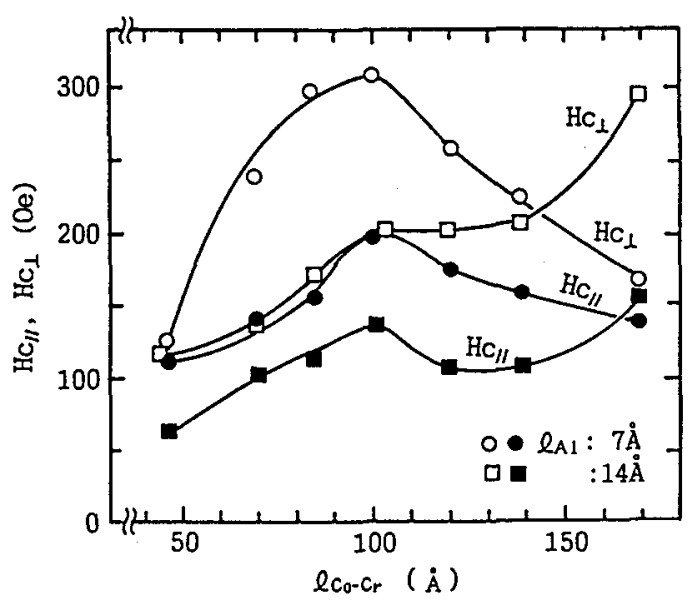

Fig.6. Dependence of coercivity $\mathrm{Hc}_{\|}$ and $\mathrm{H}_{\perp}$ on $\ell \mathrm{c}_{0}-\mathrm{c}_{r}$.

$100 \AA$. In this range, in-plane anisotropy become rather dominent. Co- $\mathrm{Cr}$ grains may become fine by compressive stress. And then $(\mathrm{Mr} / \mathrm{Ms})_{\|}$decreased steeply with further increase of $\ell c_{0}-c_{r}$. In this range, the perpendicular magnetic anisotropy was rather remarkable. In $14 \AA-A l$ layers, $(\mathrm{Mr} / \mathrm{Ms})_{\|}$is higher than that in $7 \AA-A l$ ones . The in-plane anisotropy is remarkable. The magnetic anisotropy can be controlled by $\ell_{A 1}$. This is consistent to the relationship between $\Delta \theta_{50}$ and $\ell c_{0}-c_{r}$ as shown in Fig. 4.

The dependence of the magnetoresistivity ratio $\Delta \rho$ on the external magnetic field $\mathrm{H}_{\mathrm{E}}$ are investigated, where $l \mathrm{c}_{0}-\mathrm{c}_{r}$ is $46 \AA$. The absolute value of $\Delta \rho$ was $0.05 \%$ in $14 \AA-A l$ layers for $H_{E}$ applied in the direction longitudinal to the current flow.

This was smaller than that in $7 \AA-A l$ ones.

On the other hand, the change of $\Delta \rho$ with increase of $\mathrm{H}_{\mathrm{E}}$ applied in the direction normal to the film plane was less than 0.035. This may imply that the interface between $\mathrm{Co}-\mathrm{Cr}$ layers and $\mathrm{Al}$ ones in the films become sharp with increasing $\ell_{\mathrm{Al}}$. This consideration may be also supported by the small angle X-ray diffraction diagrams as shown in Fig. 2 .

\section{CONCLUSION}

The $\mathrm{Co}_{81} \mathrm{Cr}_{19} / \mathrm{Al}$ multilayered films have been prepared on glass-slide substrates by using FTS apparatus. $\Delta \Delta_{50}$ decreased gradually with increasing $\ell \mathrm{c}_{0}-\mathrm{C}_{r}$ and $\mathrm{d}_{002}$ expanded gardually with the decrease of

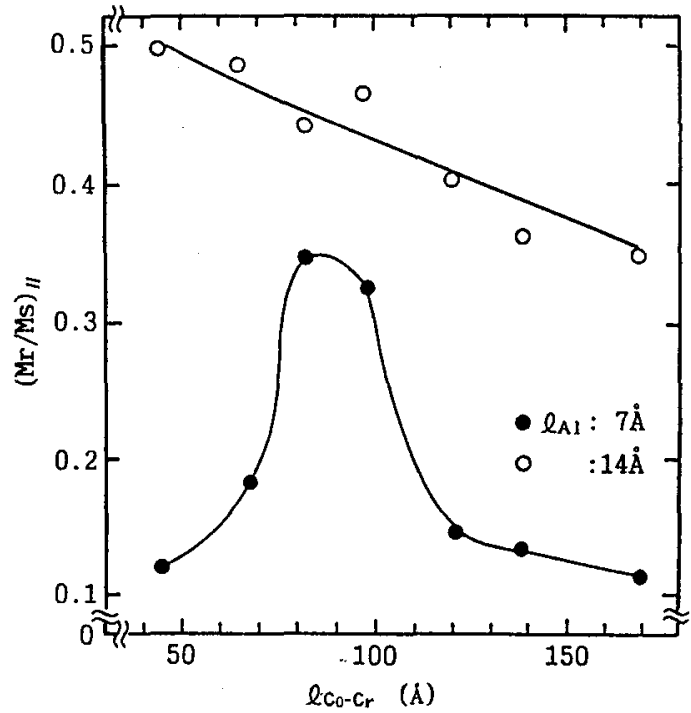

Fig.7. Dependence of in-plane squareness ratio $(\mathrm{Mr} / \mathrm{Ms})_{H}$ on $\ell \mathrm{c}_{0}-\mathrm{C}_{r}$.

$\ell c_{0}-C_{r}$, regardless of $\ell_{A 1}$. Ms depended significantly on $\ell c_{0}-c_{r}$ and $\ell_{A 1}$. Ms took maximum value of $610 \mathrm{emu} / \mathrm{cc}$ at $\ell c_{0}-C_{r}$ of 84 $\AA$ in $7 \AA-A l$ layers. Both of $\mathrm{Hc}_{/ /}$and $\mathrm{Hc}_{1}$ also depended strongly on $\ell_{c_{0}-c_{r}}$ and $\ell_{A 1}$. In $7 \AA-A 1$ layers, the perpendicular magnetic anisotropy was remarkable at $l c_{0}-c_{r}$ above $120 \AA$. In $14 \AA-A 1$ layers, the in-plane magnetic anisotropy was remarkable. The changes in Ms and Hc may be due to that the internal stress in the films induced by the different Young's modulus between $\mathrm{Co}_{81} \mathrm{Cr}_{19}$ and Al. This may be caused due to the segregation of chromium by large compressive stress occurred in the plane of Co-Cr layers.

\section{REFERENCES}

[1] S.Ono, M.Nitta and M.Naoe, IEEE Trans. Magn. MAG-25, 3872(1989).

[2] T.Mitsuzuka, A.Kamijo and H.Igarashi; J.Appl.Phys. 68(4), 1787 (1990) .

[3] R.V.Leeuwen, C.D.England, J.R.Dutcher and C.M.Falco, J.Appl.Phys. 67(9) 4910 (1990).

[4] M. Nagakubo, T.Yamanoto and M. Naoe, J.Appl.Phys. 64(10) 5751 (1988).

[5] S.Akiyama, Y.Furuto, S.Nakagawa and M.Naoe, J.Appl.Phys. 67 (9), 5181 (1990)

[6] T.Takahashi, T.Hata and M.Naoe, IEEE Trans. Magn. MAG-26, 112 (1990). 